2

\title{
Algebraic Markov Processes
}

In this chapter we describe the abstract definition and the basic facts on algebraic Markov processes (see [5]). The main goal is to show that the fundamental definitions and properties of Markov processes are easily formulated in an algebraic language suitable for the study of Markov processes appearing in quantum theory. Moreover, we discuss in detail the notion of complete positivity which turns out to be the natural generalisation of positivity for commutative (classical) case and a non-commutative version of the Feymman-Kac formula which is the basic ingredient in the construction of Markov cocycles and processes.

\section{$2.1 \quad$ Fundamental definitions}

Definition 2.1 Let $(\mathcal{A}, \varphi)$ be an algebraic probability space and let $\mathcal{B}$ be a $*_{-}$ algebra. An algebraic stochastic process on $\mathcal{A}$ with values in $\mathcal{B}$ is a family $u=\left(u_{t}\right)_{t \geq 0}$ of algebraic random variables on $\mathcal{A}$ with values in $\mathcal{B}$. The quantum stochastic process $u$ is adapted with respect to a filtration $\left(\mathcal{A}_{t]}\right)_{t \geq 0}$ if

$$
u_{t}(\mathcal{B}) \subseteq \mathcal{A}_{t]}
$$

for all $t \geq 0$.

In the remaining part of this chapter we fix the following framework:

- an algebraic probability space $(\mathcal{A}, \varphi)$ with a filtration $\left(\mathcal{A}_{t]}\right)_{t \geq 0}$ and $\mathbb{1} \in \mathcal{A}_{0]}$,

- a projective family of conditional expectations $\left(\mathbb{E}\left[\cdot \mid \mathcal{A}_{t]}\right]\right)_{t \geq 0}$,

- an adapted process $u$ with values in $\mathcal{A}_{0]}$,

- a family of conditional expectations $\left(\mathbb{E}\left[\cdot \mid u_{t}\left(\mathcal{A}_{0]}\right)\right]\right)_{t>0}$, such that $\mathbb{E}\left[\mathbb{E}\left[a \mid \mathcal{A}_{t]}\right] \mid u_{t}\left(\mathcal{A}_{0]}\right)\right]=\mathbb{E}\left[a \mid u_{t}\left(\mathcal{A}_{0]}\right)\right]$ for every $a$ in the $*$-algebra generated by $u_{s}\left(\mathcal{A}_{0]}\right)$ with $s \geq t$.

Definition 2.2 An adapted algebraic stochastic process $u$ is an algebraic Markov process, with respect to the filtration $\left(\mathcal{A}_{t]}\right)_{\iota \geq 0}$, if, for all $s, t \geq 0$ and all 
$X \in \mathcal{A}_{0]}$, we have

$$
\mathbb{E}\left[u_{t+s}(X) \mid \mathcal{A}_{s]}\right]=\mathbb{E}\left[u_{t+s}(X) \mid u_{s}\left(\mathcal{A}_{0]}\right)\right] .
$$

An algebraic Markov process is covariant or homogeneous if, for all $s, t \geq 0$ and all $a \in \mathcal{A}_{0]}$, we have

$$
u_{t}\left(\mathbb{E}\left[u_{s}(a) \mid \mathcal{A}_{0]}\right]\right)=\mathbb{E}\left[u_{t+s}(a) \mid \mathcal{A}_{t]}\right] .
$$

Example 2.1 To illustrate the above definition we show that an homogeneous classical Markov process can be considered as a covariant quantum Markov process.

Let $x=\left(x_{t}\right)_{t \geq 0}$ be a classical (adapted) Markov process with values in a measurable space $(E, \mathcal{E})$, initial law $\mu$ and transition probability function

$$
P: D \times E \times \mathcal{E} \rightarrow \mathbb{R},
$$

where $D=\{(s, t) \in[0,+\infty) \mid 0 \leq s \leq t\}$. Suppose that, for every $(s, t) \in D$, $x \in E$ and every $A \in \mathcal{E}$ such that $\mu(A)=0$ we have $P(s, t, x, A)=0$. We consider the canonical realization on the classical probability space $(\Omega, \mathcal{F}, \mathbb{P})$ where

$$
\Omega=\prod_{t \geq 0} E, \quad \mathcal{F}=\otimes_{t \geq 0} \mathcal{E},
$$

and $\mathbb{P}$ is the probability measure on $\mathcal{F}$ defined by

$$
\begin{aligned}
\mathbb{E}\left[f\left(x_{t_{1}}, \ldots, x_{t_{n}}\right)\right] & =\int_{E} d \mu(z) \int_{E} P\left(0, t_{1}, z, d z_{1}\right) \int_{E} P\left(t_{1}, t_{2}, z_{1}, d z_{2}\right) \ldots \\
& \cdots \int_{E} P\left(t_{n-1}, t_{n}, z_{n-1}, d z_{n}\right) f\left(z_{1}, \ldots, z_{n}\right)
\end{aligned}
$$

where $0 \leq t_{1}<t_{2}<\ldots<t_{n}$. Consider the filtration $\left(\mathcal{F}_{t]}\right)_{t \geq 0}$ given by

$$
\mathcal{F}_{t\}}=\prod_{0 \leq s \leq t} \mathcal{E}
$$

Consider a quantum probability space $(\mathcal{A}, \varphi)$ with a filtration $\left(\mathcal{A}_{t]}\right)_{t \geq 0}$ where

$$
\begin{aligned}
\mathcal{A}= & L^{\infty}(\Omega, \mathcal{F} ; \mathbb{C}), \quad \mathcal{A}_{t]}=L^{\infty}\left(\Omega, \mathcal{F}_{t]} ; \mathscr{C}\right), \\
& \varphi\left(f\left(x_{t_{1}}, \ldots, x_{t_{n}}\right)\right)=\mathbb{E}\left[f\left(x_{t_{1}}, \ldots, x_{t_{n}}\right)\right] .
\end{aligned}
$$

The classical process $x$ defines a family of ${ }^{*}$-homomorphisms

$$
u_{t}: \mathcal{A}_{0]} \rightarrow \mathcal{A}_{t]}, \quad u_{t}(f)=f\left(x_{t}\right) .
$$

Therefore the classical Markov property

$$
\mathbb{E}\left[f\left(x_{t+s}\right) \mid \mathcal{F}_{s}\right]=\mathbb{E}\left[f\left(x_{t+s}\right) \mid x_{s}\right]
$$


for all $s, t \geq 0$ and all $f \in \mathcal{A}_{0]}$ is immediately translated, in the algebraic language, into the identity (2.1).

The Markov process is time homogeneous if and only if

$$
\int_{E} f(y) P(s, t+s, x, d y)=\int_{E} f(y) P(0, t, x, d y)
$$

for all $s, t \geq 0$, and all $f \in \mathcal{A}_{0]}$. Now (2.2) can be easily understood in vicw of the following correspondence table between the quantum and classical case

$$
\begin{aligned}
\text { quantum } & \longleftrightarrow & \text { classical } \\
\mathbb{E}\left[u_{t+s}(f) \mid \mathcal{A}_{t]}\right] & \longleftrightarrow & \int_{E} f(y) P\left(t, t+s, x_{t}, d y\right) \\
\mathbb{E}\left[u_{s}(f) \mid \mathcal{A}_{0]}\right] & \longleftrightarrow & \int_{E} f(y) P(0, s, x, d y) \\
u_{t}\left(\mathbb{E}\left[u_{s}(f) \mid \mathcal{A}_{0]}\right]\right) & \longleftrightarrow & \int_{E} f(y) P\left(0, s, x_{t}, d y\right) .
\end{aligned}
$$

Proposition 2.3 The following conditions are equivalent:

1. $u$ is an homogeneous algebraic Markov process,

2. for all $n \geq 1$ and all $a_{1}, \ldots, a_{n} \in \mathcal{A}_{0]}, 0<t_{1}<\ldots<t_{n}, s \geq 0$ we have

$$
\begin{aligned}
\mathbb{E}\left[u_{t_{1}+s}\left(a_{1}\right)\right. & \left.\ldots u_{t_{n}+s}\left(a_{n}\right) \mid \mathcal{A}_{s]}\right] \\
& =\mathbb{E}\left[u_{t_{1}+s}\left(a_{1}\right) \ldots u_{t_{n}+s}\left(a_{n}\right) \mid u_{s}\left(\mathcal{A}_{0]}\right)\right], \\
u_{\ell_{1}}\left(\mathbb{E}\left[u_{s}\left(a_{1}\right) \mid \mathcal{A}_{0]}\right]\right) & \left.=\mathbb{E}\left[u_{t_{1}+s}\left(a_{1}\right) \mid \mathcal{A}_{t_{1}}\right]\right] .
\end{aligned}
$$

Proof. Clearly it suffices to prove that, if $u$ is an homogeneous quantum Markov process, then the first identity of condition 2 holds. Wo will consider the case $n=2$ for simplicity. Using the Markov, covariance and *-honomorphism property of $u$ we can show that the conditional expectation

$$
\left.\mathbb{E}\left[u_{t_{1}+s}\left(a_{1}\right) u_{t_{2}+s}\left(a_{2}\right) \mid \mathcal{A}_{s}\right]\right]
$$

is equal to

$$
\begin{aligned}
& \left.\mathbb{E}\left[u_{t_{1}+s}\left(a_{1}\right) \mathbb{E}\left[u_{t_{2}+s}\left(a_{2}\right) \mid \mathcal{A}_{t_{1}+s}\right] \mid \mathcal{A}_{s}\right]\right] \\
= & \mathbb{E}\left[u_{l_{1}+s}\left(a_{1}\right) u_{l_{1}+s}\left(\mathbb{E}\left[u_{t_{2}-l_{1}}\left(a_{2}\right) \mid \mathcal{A}_{0]}\right]\right) \mid \mathcal{A}_{s]}\right] \\
= & \mathbb{E}\left[u_{t_{1}+s}\left(a_{1} \mathbb{E}\left[u_{t_{2}-t_{1}}\left(a_{2}\right) \mid \mathcal{A}_{0]}\right]\right) \mid \mathcal{A}_{s]}\right] \\
= & \mathbb{E}\left[u_{t_{3}+s}\left(a_{1} \mathbb{E}\left[u_{t_{2}-t_{1}}\left(a_{2}\right) \mid \mathcal{A}_{0]}\right]\right) \mid u_{s}\left(\mathcal{A}_{0]}\right)\right] \\
= & \mathbb{E}\left[u_{t_{1}+s}\left(a_{1}\right) u_{t_{1}+s}\left(\mathbb{E}\left[u_{t_{2}-t_{1}}\left(a_{2}\right) \mid \mathcal{A}_{0]}\right]\right) \mid u_{s}\left(\mathcal{A}_{0\}}\right)\right] \\
= & \mathbb{E}\left[u_{t_{1}+s}\left(a_{1}\right) \mathbb{E}\left[u_{t_{2}+s}\left(a_{2}\right) \mid \mathcal{A}_{\left.t_{1}+s\right]}\right] \mid u_{s}\left(\mathcal{A}_{0]}\right)\right] \\
= & \mathbb{E}\left[u_{l_{1}+s}\left(a_{1}\right) u_{t_{2}+s}\left(a_{2}\right) \mid u_{s}\left(\mathcal{A}_{0]}\right)\right] .
\end{aligned}
$$


This completes the proof.

The following proposition shows that, as in classical probability, one can associate a semigroup to an algebraic Markov process.

Proposition 2.4 Let $u$ be a covariant algebraic Markov process. For all $t \geq 0$ define the map

$$
\mathcal{T}_{t}: \mathcal{A}_{0\}} \rightarrow \mathcal{A}_{0 !}, \quad \mathcal{T}_{t}(a)=\mathbb{E}\left[u_{t}(a): \mathcal{A}_{0\}}\right]
$$

Then $\mathcal{T}=\left(\mathcal{T}_{t}\right)_{t \geq 0}$ is a semigroup of operators in $\mathcal{A}_{0}$ with the following propertics

1. for every integer $n \geq 1$ and every fomily $a_{1}, \ldots, a_{n}, b_{1}, \ldots, b_{n}$ of elements of $\mathcal{A}_{0 !}$ we have

$$
\sum_{p, q=1}^{n} b_{p}^{*} T_{i}\left(a_{p}^{*} a_{q}\right) b_{q} \geq 0
$$

for every $t \geq 0$,

2. $\mathcal{T}_{t}(\mathbb{1})=\mathbb{1}$ for every $t \geq 0$.

Proof. $\mathcal{T}$ is a semigroup in fact, for crery $t, s \geq 0$ and $a \subset \mathcal{A}$, wo have,

$$
\begin{aligned}
& \text { (projectivity) } \\
& \text { (covariance) }
\end{aligned}
$$$$
T_{l+s}(a) \quad \mathbb{E}\left[u_{t+s}(a) \mid \mathcal{A}_{0}\right]
$$$$
=\mathbb{E}\left[\mathbb{E}\left[u_{t+s}(a): \mathcal{A}_{l]}\right] \mid \mathcal{A}_{0 \mid}\right]
$$$$
=\mathbb{E}\left[u_{i}\left(\mathbb{E}\left[u_{s}(a) \mid \mathcal{A}_{0]}\right]\right) \mid \mathcal{A}_{0]}\right]
$$$$
=\mathcal{T}_{t}\left(T_{s}(a)\right)
$$

Clearly property 2 holds since $\mathbb{1}$ belongs to $\mathcal{A}_{0]}$ and both $u_{l}$ and the conditional expectation preservo 11 .

Finally for every integer $n \geq 1$ and every family $a_{1}, \ldots, a_{n}, b_{1}, \ldots, b_{n}$ of clements of $\mathcal{A}_{0]}$ the left-hand side of $(2.4)$ is equal to

$$
\begin{aligned}
\sum_{p, q=1}^{n} b_{p}^{*} \mathbb{E}\left[u_{t}\left(a_{p}^{*} a_{q}\right) \mid \mathcal{A}_{0]}\right] b_{q} \\
=\sum_{p, q=1}^{n} \mathbb{E}\left[b_{p}^{*}, u_{t}\left(a_{p}^{*}\right) u_{t}\left(a_{q}\right) b_{q} \mid \mathcal{A}_{0 !}\right] \\
=\mathbb{E}\left[\left(\sum_{p=1}^{n} u_{t}\left(a_{p}\right) b_{p}\right)^{*}\left(\sum_{q=1}^{n} u_{t}\left(a_{q}\right) b_{q}\right) \mid \mathcal{A}_{0\}}\right]
\end{aligned}
$$

which is clearly a positive operator because of property 1 of conditional expectation. This completes the proof.

Property 1 of algebraic Markov semigroup is called complete positivity and plays a key role in our exposition. As we have scon in the proof of Proposition 2.4 , it follows from complete positivity of conditional expectation. More 
general conditional expectations in quantum probability (see [3], [77]) are also completely positive. Therefore complete positivity is the proper generalization of positivity in the non-commutative framework.

The next section will be devoted to a detailed study of this property and its connections with positivity.

\subsection{Completely positive linear maps}

Let $\mathcal{A}$ and $\mathcal{B}$ be two $*$-algebras with unit. We denote by $\mathbb{1}$ the unit of both since each time it will be clear from the context to which algebra it belongs.

The obvious generalization of the notion of positivity for classical (sub) Markov operators is too weak when $\mathcal{A}$ and $\mathcal{B}$ are non commutative. Proposition 2.4 motivates the introduction of the following stronger notion of positivity.

a. inition 2.5 The linear map $T: \mathcal{A} \rightarrow \mathcal{B}$ is called:

1. $n$-positive if for every family $a_{1}, \ldots, a_{n}$ of elements of $\mathcal{A}$ and every family $b_{1}, \ldots, b_{n}$ of elements of $\mathcal{B}$ we have

$$
\sum_{p, q=1}^{n} b_{p}^{*} T\left(a_{p}^{*} a_{q}\right) b_{q} \geq 0,
$$

2. completely positive if it is $n$ positive for every integer $n \geq 1$.

The following fact is obvious.

Proposition 2.6 Let $T: \mathcal{A} \rightarrow \mathcal{B}$ be $a^{*}$-homomorphism. Then $T$ is completely positive.

1 .

In the remaining part of this section $\mathcal{A}$ and $\mathcal{B}$ will be $C^{*}$-algebras with unit

Note that, for every integer $n \geq 1$, the algelbraic tensor product $*$-algebras $\mathcal{A} \propto \mathcal{M}_{n}$ and $\mathcal{B} \propto \mathcal{M}_{n}$ can be represented as the $*$-algebras of $n \times n$ matrices with entries in $\mathcal{A}$ and $\mathcal{B}$ respectively. Every clement $x$ of $\mathcal{A} \times \mathcal{M}_{n}$ can be written in the form

$$
x=\sum_{1 \leq i, j \leq n} x_{i j} \otimes E_{i j}
$$

where $E_{i j}$ denotes the $n \times n$ matrix with all the entries equal to 0 except the $i j$-th which is equal to 1 .

Given a linear map $T: \mathcal{A} \rightarrow \mathcal{B}$ we can define linear maps $T_{n}: \mathcal{A} \otimes \mathcal{M}_{n} \rightarrow$ $\mathcal{B} \otimes \mathcal{M}_{n}$ by

$$
T_{n}\left(a \otimes E_{i j}\right)=T(a) \otimes E_{i j}
$$

In order to give a useful condition equivalent to complete positivity by means of the maps $T_{n}$ we prove a simple fact on positive elements of $\mathcal{A} \otimes \mathcal{M}_{n}$ in the case when $\mathcal{A}$ is a $C^{*}$-algebra. 
Proposition 2.7 Let $\mathcal{A}$ be a $C^{*}$-algebra and let $x$ be an element of $\mathcal{A} \otimes_{n}$. The following conditions are equivalent:

1. $x$ is positive,

2. $x$ is a finite sum of matrices of the form

$$
\sum_{1 \leq i, j \leq n} a_{i}^{*} a_{j} \ltimes E_{i j}
$$

with $a_{1}, \ldots, a_{n} \in \mathcal{A}$,

3. for all $a_{1}, \ldots, a_{n} \in \mathcal{A}$ we have

$$
\sum_{1 \leq i, j, \leq n} a_{i}^{*} x_{i j} a_{j} \geq 0
$$

Proof. 1 implies 2. In fact, since $x$ is positive, it can be written in the form $y^{*} y$ with $y \in \mathcal{A} \otimes \mathcal{M}_{n}$ (see, for example, [21], Th. 2.2.10). Writing $y$ in the form (2.5) we have

$$
x=\sum_{\ell=1}^{n} \sum_{1 \leq i, j \leq n} y_{\ell i}^{*} y_{\ell j} \alpha E_{i j} .
$$

2 obviously implies 3.

3 implies 1. By representing $\mathcal{A}$ as a sub- $C^{*}$-algebra of the algebra of all bounded operators on a Hilbert space $\mathcal{H}$ (see [21] Th. 2.1.10 p. 60) and decomposing $\mathcal{H}$ into cyclic orthogonal subspaces we may suppose that there exists a cyclic vector $u$ for the representation. Condition 3 then implies then the inequality

$$
\sum_{i, j}\left\langle a_{i} u, r_{i j} a_{j} u\right\rangle \geq 0
$$

Therefore, since the vector $u$ is cyclic, we have

$$
\sum_{i, j}\left\langle v_{i}, x_{i j} v_{j}\right\rangle \geq 0
$$

for all $v_{1}, \ldots, v_{n} \in \mathcal{H}$. This completes the proof.

We are now in a position to prove the following

Proposition 2.8 Let $\mathcal{A}, \mathcal{B}$ be $C^{*}$-algebras and let $T: \mathcal{A} \rightarrow \mathcal{B}$ be a linear map. The following conditions are equivalent:

1. $T$ is completely positive,

2. for every integer $n \geq 1$ the map $T_{n}$ defined by (2.6) is positive. 
Proof. The second condition implies the first by parts 2 and 3 of Proposition 2.7. Since the operator $x=\sum_{i, j} a_{i}^{*} a_{j} \otimes E_{i j}$ in $\mathcal{A} \otimes \mathcal{M}_{n}$ is positive, we have that $T_{n}(x)=\sum_{i, j} T\left(a_{i}^{*} a_{j}\right) \otimes E_{i j}$ is positive.

Conversely the first condition implies that $\sum_{i, j} T\left(a_{i}^{*} a_{j}\right) \otimes E_{i j}$ is positive. Therefore $T_{n}$ is positive because of the equivalence of conditions 1 and 2 of Proposition 2.7.

Proposition 2.9 Let $T: \mathcal{A} \rightarrow \mathcal{B}$ be a linear map where $\mathcal{B}$ is the $C^{*}$-algebra $\mathcal{B}(\mathcal{K})$ of all bounded operators on a Hilbert space $\mathcal{K}$. Then $T$ is completely positive if and only if for every integer $n \geq 1$ and every $a_{1}, \ldots, a_{n} \in \mathcal{A}$, $u_{1}, \ldots, u_{n} \in \mathcal{K}$

$$
\sum_{1 \leq i, j \leq n}\left\langle u_{i}, T\left(a_{i}^{*} a_{j}\right) u_{j}\right\rangle \geq 0
$$

Proof. Notice that the $C^{*}$-algebra $\mathcal{B} \otimes \mathcal{M}_{n}$ can be represented as the $C^{*}$-algebra of all bounded operators on the $n$-fold direct sum $\mathcal{K}(\ldots) \mathcal{K}$. Therefore the above condition is clearly equivalent to positivity of the map $T_{n}$ on $\mathcal{A} \otimes \mathcal{M}_{n}$ for cvery integer $n \geq 1$.

The following counterexample, essentially due to W.B. Arveson [12], shows the existence of positive maps that are not completely positive. Let $n \geq 2$ be an integer and let both $\mathcal{A}$ and $\mathcal{B}$ be the *-algebra $\mathcal{M}_{n}$. Consider the positive linear map

$$
T: \mathcal{M}_{n} \rightarrow \mathcal{M}_{n}, \quad T(a)=a^{t}
$$

( $a^{t}$ denoting the transpose matrix). We will prove that $T$ is not 2-positive. Let $b$ be the $2 \times 2$ matrix with entries in $\mathcal{M}_{n}$ having the matrix $E_{11}$ as 11-th entry, $E_{1 n}$ as 12-th entry, $E_{n 1}$ as 21-th entry, $E_{n n}$ as 22-th entry. For example, when $n=2$, we have

$$
b=\left(\begin{array}{cccc}
1 & 0 & 0 & 1 \\
0 & 0 & 0 & 0 \\
0 & 0 & 0 & 0 \\
1 & 0 & 0 & 1
\end{array}\right), \quad T_{2}(b)=\left(\begin{array}{cccc}
1 & 0 & 0 & 0 \\
0 & 0 & 1 & 0 \\
0 & 1 & 0 & 0 \\
0 & 0 & 0 & 1
\end{array}\right) .
$$

It is easy to check that the matrix $b / 2$ is a self-adjoint projection. Thus it is positive. However the $2 n \times 2 n$ real matrix $T_{n}(b)$ is not positive because its elements do not satisfy the inequality $\left|x_{i j}\right|^{2} \leq x_{i i} x_{j j}$ for $i=n, j=n+1$.

Other counterexamples of maps which are $n$-positive but not $(n+1)$-positive for an arbitrary integer $n$ can be found in [29].

We deduce now two useful properties of 2-positive maps.

Proposition 2.10 Let. $\mathcal{A}, \mathcal{B}(\mathcal{B} \subseteq \mathcal{B}(\mathcal{K}))$ be $C^{*}$-algebras with unit and let $T$ : $\mathcal{A} \rightarrow \mathcal{B}$ with be a 2-positive map. Then:

1. if $T(\mathbb{1})$ is invertible in $\mathcal{B}$ then for all $a \in \mathcal{A}$ we have the Schwarz inequality

$$
T\left(a^{*}\right)(T(\mathbb{1}))^{-1} T(a) \leq T\left(a^{*} a\right),
$$


2. for all $a \in \mathcal{A}$ we have the incquality

$$
T\left(a^{*}\right) T(a) \leq \| T(\mathbb{I}): T\left(a^{*} a\right),
$$

3. $T$ is continuous and

$$
\|T\|=T(1)
$$

Proof. The operator in $\mathcal{B}(\mathcal{K}) \times \mathcal{M}_{2}$

$$
\left(\begin{array}{cc}
T\left(a^{*} a\right) & T\left(a^{*}\right) \\
T(a) & T(\mathbb{1})+\varepsilon \mathbb{1}
\end{array}\right)=T_{2}\left(\begin{array}{cc}
a^{*} a & a^{*} \\
a & \mathbb{1}
\end{array}\right)+\left(\begin{array}{cc}
0 & 0 \\
0 & -1 \mathbb{1}
\end{array}\right)
$$

is positive for every $\varepsilon>0$. Hence, for "very $u, l^{\prime} \in \mathcal{\Lambda}$, we have

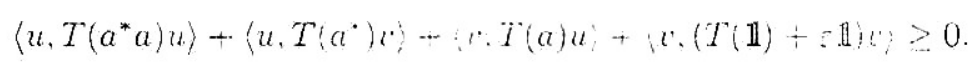

The operator $T(\mathbb{1})+\varepsilon \mathbb{1}$ has a bounded incerse. Taking $r=-(T(\mathbb{1})+\varepsilon \mathbb{1})^{-1} \% \psi_{n}$ u we have the ineciuality

$$
\left\langle u \cdot T\left(a^{*}\right)(T(\mathbb{1})+\equiv \mathbb{1})^{-1} T(a) u\right\rangle \leq\left\langle u \cdot T\left(a^{*} a\right) u\right\rangle
$$

for all $u \in \mathcal{K}$. If $T(\mathbb{1})$ is invertible in $\mathcal{B}$ then, by letting $\varepsilon$ teud to () we obtain the inequality (2.8).

In any case, since $\mathbb{1} \leq \| T(\mathbb{1})+\varepsilon \mathbb{1}(T(\mathbb{1})+\varepsilon \mathbb{1})^{-1}$, we find the inequality

$$
T\left(a^{*}\right) T(a) \leq\|T(\mathbb{1})+\varepsilon \mathbb{1}\| T\left(a^{*}\right)(T(\mathbb{1})+\varepsilon \mathbb{1})^{-1} T(a) \leq \| T(\mathbb{1})+\varepsilon \mathbb{1} T\left(a^{*} a\right) .
$$

Therefore, letting $\varepsilon$ tend to 0 , we obtain (2.9).

The inequality (2.9) and the property $\mid x^{*} \cdot x^{2}=\mu^{2}$ of $C^{*}$ norms immeilately yield

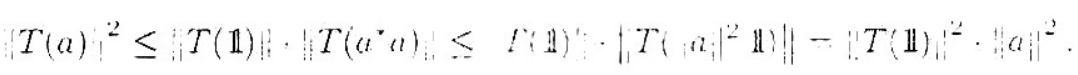

Thin completes the proof.

The following results show, in particular, that a positive linear map $T: \mathcal{A} \rightarrow$, $\mathcal{B}$ is in fact completely positive when at least one of the $("$ algebras $\mathcal{A}$ and $\mathcal{B}$ is commutative. We refer to [12], 82 tior the prons.

Theorem 2.11 (Arotson) Let $B$ be a commutative $C^{*}$-slgebra. Then exery positive linear map $T: \mathcal{A} \rightarrow \mathcal{B}$ is completely positive.

Theorem 2.12 (Stinespring) Let $\mathcal{A}$ be a commutatue $C^{*}$-algebru. Then every positive linear map $T: \mathcal{A} \rightarrow \mathcal{B}$ is completely positive.

The following simple properties of completcly positive maps tum out to be useful.

Proposition 2.13 Let $T: \mathcal{A} \rightarrow \mathcal{B}$ and $S: \mathcal{A} \rightarrow \mathcal{B}$ be two completely positive linear maps. Then the map $S+T$ completely positive. 
Proof. Obvious.

Proposition 2.14 Let $\mathcal{A}_{1}, \mathcal{A}_{2}, \mathcal{A}_{3}$ be $C^{*}$-algebras and $T: \mathcal{A}_{1} \rightarrow \mathcal{A}_{2}, S: \mathcal{A}_{2} \rightarrow$ $\mathcal{A}_{3}$ be two completely positive linear maps. Then the linear map $S \circ T: \mathcal{A}_{1} \rightarrow \mathcal{A}_{3}$ is completely positive.

Proof. It suffices to notice that, for every integer $n \geq 1$, the map $(S \circ T)_{n}$ : $\mathcal{A}_{1} \otimes \mathcal{M}_{n} \rightarrow \mathcal{A}_{3} \propto \mathcal{M}_{n}$ coincides with the composition $S_{n} \circ T_{n}$.

Proposition 2.15 Let $\mathcal{K}$ be a Hilbert space and let $\left(T_{m}\right)_{m \geq 1}$ be a sequence of completely positive lincar maps $T_{m}: \mathcal{A} \rightarrow \mathcal{B}(\mathcal{\Lambda})$. Suppose that, for coery $a \in \mathcal{A}$, the sequence $\left(T_{m}(a)\right)_{m \geq 1}$ converges weakly. Then the linear map $T^{\prime}: \mathcal{A} \rightarrow \mathcal{B}(\mathcal{K})$ defined by

$$
T(a)=\lim _{m \rightarrow \infty} T_{m}(a)
$$

in completely positive.

Proof. By Proposition 2.9 it suffices to note that

$$
\sum_{1 \leq i, j \leq n}\left\langle u_{i}, T\left(a_{i}^{*} a_{j}\right) u_{j}\right\rangle=\lim _{m \rightarrow \infty} \sum_{1 \leq i, j \leq n}\left\langle u_{i}, T_{m}\left(a_{i}^{*} a_{j}\right) u_{j}\right\rangle \geq 0 .
$$

for every integer $n \geq 1$, every $u_{1}, \ldots, u_{n} \in \mathcal{A}$ and wery $a_{1}, \ldots, a_{n} \in \mathcal{A}$.

W.F. Stinespring proved in [82] the following characterization of completer positive maps.

Theorem 2.16 (Stinespring) Let $\mathcal{B}$ be a sub) $C^{*}$-algebra of the algebra of wh bounded operators on a Hilbert space $\mathcal{H}$ and let. $\mathcal{A}$ be a $C^{*}$-algebra with wnit. 1 linear map $T: \mathcal{A} \rightarrow \mathcal{B}$ is completely positive if and only if it has the form

$$
T(a)=V^{*} \pi(a) V
$$

where $(\pi, \mathcal{K})$ is a representation of $\mathcal{A}$ on $\mathcal{K}$ for some Hilbert space $\mathcal{K}$, and $\mathcal{V}$ is a bounded operator from $\mathcal{H}$ to $\mathcal{K}$.

Proof. Let $T$ be a linear map of the form $(2.10)$ and let $\left(a_{i j}\right)_{1 \leq i_{1}, j \leq . .}$ be an positive matrix in $\mathcal{A} \otimes \mathcal{M}_{n}$. For all vectors $\left(u_{j}\right)_{1 \leq j \leq n}$ in $\mathcal{H}$ we have then

$$
\sum_{i, j}\left\langle u_{i}, T\left(a_{i j}\right) u_{j}\right\rangle=\sum_{i, j}\left\langle V u_{i}, \pi\left(a_{i j}\right) V u_{j}\right\rangle \geq 0
$$

because $\pi$ is completely positive by Proposition 2.6 .

Conversely suppose that $T$ is completely positive and consider the vector space $\mathcal{A} \otimes \mathcal{H}$, the algebraic tensor product of $\mathcal{A}$ and $\mathcal{H}$. On this space we define the bilinear form $(\cdot, \cdot)$

$$
(x, y)=\sum_{i, j}\left\langle u_{i}, T\left(a_{i}^{*} b_{j}\right) v_{j}\right\rangle
$$


for $x=\sum_{i} a_{i} \otimes u_{i}$ and $y=\sum_{j} b_{j} \bowtie v_{j}$ in $\mathcal{A} \times \mathcal{H}$. Since $T$ is completely positive we have

$$
(x, x)=\sum_{i, j}\left\langle u_{i} \cdot T\left(a_{i}^{*} a_{j}\right) u_{j}\right\rangle \geq 0
$$

for all $x \in \mathcal{A} \times \mathcal{H}$. Hence the bilinear form $(\cdot, \cdot)$ is positive. Consider the algebra homomorphism $\pi_{0}$ defined on $\mathcal{A}$ with values in linear transfornations in $\mathcal{A} \times \mathcal{H}$

$$
\pi_{0}(a)\left(\sum_{i} a_{i} \propto u_{i}\right)=\sum_{i}\left(a a_{i}\right) \propto u_{i} .
$$

Notice that, for all $x, y$ as above, we have

$$
\left(x \cdot \pi_{0}(a) y\right)=\left(\pi_{0}\left(a^{*}\right) x, y\right) .
$$

It follows that, for every $x \in \mathcal{A} \& \mathcal{H}$, the linear map

$$
\omega: \mathcal{A} \rightarrow \mathbb{Q}, \quad \omega(a)=\left(x, \pi_{0}(a) x\right)
$$

is a positive linear functional on $\mathcal{A}$. Therefore we have (see [21] Prop. 2.3.11)

$$
\left\|\pi_{0}(a) x\right\|^{2}=\left(x, \pi_{0}\left(a^{*} a\right) x\right) \leq a^{*} a\left\|\omega(\mathbb{1})=a_{i}^{2}: x^{2}\right\|^{2} .
$$

Let $\mathcal{N}$ be the linear subspace of vectors $x$ in $\mathcal{A} \bowtie \mathcal{H}$ such that $(x . x)=0$. Since $\mathcal{V}$ is invariant under $\pi_{0}(a)$ for every $a \in \mathcal{A}$ because of (2.11). we can consider the quotient pre-Hibert space $\mathcal{A} \& \mathcal{H} / \mathcal{N}$ defining the pre-scalar product on $\mathcal{A}, \mathcal{H} / \mathcal{N}$ by

$$
(x+\mathcal{N}, x+\mathcal{N})=(x, x) .
$$

Let $\mathcal{K}$ be the Hilbert space obtained by completion. By the above construction the *-homomorphism $\pi_{0}$ extends to a representation $\pi$ of $\mathcal{A}$ into $B(K)$ such that

$$
\pi(a)(x+1)=\pi(a) x+\mathcal{N}
$$

for $a \subset \mathcal{A}$ and $x \in \mathcal{A}, \mathcal{H}$. Consider the lincar operator $V: \mathcal{H} \rightarrow \mathcal{K}$

$$
V_{i t}=1 \cdot:-V^{*}
$$

This operator is bounded because of the inequality

$$
\|V u\|^{2}=\{u T T(1) u\rangle \leq\|T(1)\| u \|^{2} .
$$

A straightforward computation yields (2.10).

Definition 2.17 A pair $(\pi, V)$ satisfying (2.10) is called a Stinespring reprosentation of the completely positive map $T$. It is called a minimal Stincspring representation if the Hilbert space $A$ coincides with the closure of the vecto? subspace generated by

$$
\{\pi(a) V u: a \in \mathcal{A}, u \in \mathcal{H}\}
$$


In other words a pair $(\pi, V)$ is a minimal Stinespring representation if the set (2.12) is total in $\mathcal{K}$.

Every completely positive map admits a minimal Stinespring representation. In fact, with the notation of the proof of Theorem 2.16, it suffices to consider as Hilbert space $\mathcal{K}$ the closure $\mathcal{K}_{1}$ of the vector space generated by (2.12). The restriction $\pi_{1}$ of $\pi$ to $\mathcal{K}_{1}$ also satisfies (2.10).

The minimal Stinespring representation is unique in the following sense

Proposition 2.18 Let $\pi_{1}$ and $\pi_{2}$ be two representations of $\mathcal{A}$ on Hilbert spaces $\mathcal{K}_{1}$ and $\mathcal{K}_{2}$ and let $V_{i}: \mathcal{H} \rightarrow \mathcal{K}_{i}$ be two bounded operators such that

$$
\left\{\pi_{i}(a) V_{i} u \mid a \in \mathcal{A}, u \in \mathcal{H}\right\},
$$

is total $\mathcal{K}_{i}$ for $i=1,2$ and such that

$$
T(a)=V_{i}^{*} \pi(a) V_{i}
$$

for $i=1,2$. Then there exists a unitary map $U: \mathcal{K}_{1} \rightarrow \mathcal{K}_{2}$ such that

$$
U V_{1}=V_{2}, \quad U \pi_{1}(a)=\pi_{2}(a) U
$$

for all $a \in \mathcal{A}$.

Proof. Let $U: \mathcal{K}_{1} \rightarrow \mathcal{K}_{2}$ be the densely dofined lincar map defined by

$$
U\left(\sum_{j=1}^{n} \pi_{1}\left(a_{j}\right) V_{1} u_{j}\right)=\sum_{j=1}^{n} \pi_{2}\left(a_{j}\right) V_{2} u_{j}
$$

for every integer $n \geq 1$ and $a_{1}, \ldots, a_{n} \in \mathcal{A}, u_{1}, \ldots, u_{n} \in \mathcal{H}$. A straightforward computation shows that

$$
\left(U \pi_{1}(b) V_{1} v, U \pi_{1}(a) V_{1} u\right)_{2}=\left\langle v, T\left(b^{*} a\right) u\right\rangle=\left(V_{1} v, \pi_{1}\left(b^{*} a\right) V_{1} u\right)_{1},
$$

where $(\cdot, \cdot)_{j}$ denotes the scalar product in $\mathcal{K}_{j}$, for all $a, b \in \mathcal{A}$ and $u, v \in \mathcal{H}$. Therefore $U$ is an isometry and can be extended to $\mathcal{K}_{1}$ by an obvious density argument. In a similar way one can prove that also $U^{*}: \mathcal{K}_{2} \rightarrow \mathcal{K}_{1}$ is an isometry. Thus $U$ is unitary. Finally, since

$$
U V_{1} u=U \pi_{1}(\mathbb{1}) V_{1} u=\pi_{2}(\mathbb{1}) V_{2} u=V_{2} u, \quad U \pi_{1}(a) V_{1} u=\pi_{2}(a) V_{2} u
$$

for every $u \in \mathcal{H},(2.13)$ follows.

We finish this section by proving K. Kraus' characterisation [63] of $\sigma$-weakly continuous (i.e. normal) completely positive maps.

Lemma 2.19 Let $\mathcal{A}$ and $\mathcal{B}$ be two von Neumann algebras of operators on Hilbert spaces $\mathcal{H}$ and $\mathcal{K}$. A normal completely positive map $T: \mathcal{A} \rightarrow \mathcal{B}$ can be written in the form

$$
T(a)=V^{*} \pi(a) V
$$

where $V$ is a bounded operator from $\mathcal{K}$ to a Hilbert space $\mathcal{K}_{1}$ and $\pi$ is a normal representation of $\mathcal{A}$ in $\mathcal{B}\left(\mathcal{K}_{1}\right)$. 
Proof. Let $(\pi, V)$ be a minimal Stinespring representation of $T$ with $V: \mathcal{K} \rightarrow$ $\mathcal{K}_{1}$. We check that $\pi$ is normal.

Let $\left(x_{\alpha}\right)_{\alpha}$ be a non-decreasing net of elements of $\mathcal{A}$ converging to $x(x \in \mathcal{A})$ in the $\sigma$-weak topology. For all vectors $u, v \in \mathcal{K}$ and operators $a, b \in \mathcal{A}$ we have

$$
\begin{aligned}
\lim _{\alpha}\left\langle\pi(b) V v, \pi\left(x_{\alpha}\right) \pi(a) V u\right\rangle & =\lim _{\alpha}\left\langle V v, \pi\left(b^{*} x_{\alpha} a\right) V u\right\rangle \\
& =\lim _{\alpha}\left\langle v, T\left(b^{*} x_{\alpha} a\right) u\right\rangle \\
& =\left\langle v, T\left(b^{*} x a\right) u\right\rangle \\
& =\langle\pi(b) V v, \pi(x) \pi(a) u\rangle
\end{aligned}
$$

because $T$ is normal. Thus $\pi$ is normal by Proposition 1.154 .

Theorem 2.20 (Kraus) Let $\mathcal{A}$ be a von Neumann algebra of operators on a Hilbert space $\mathcal{H}$ and let $\mathcal{K}$ be another Hilbert space. A linear map $T: \mathcal{A} \rightarrow \mathcal{B}(\mathcal{K})$ is normal and completely positive if and only if it can be represented in the form

$$
T(a)=\sum_{j=1}^{\infty} V_{j}^{*} a V_{j}
$$

where $\left(V_{j}\right)_{j=1}^{\infty}$ are bounded operators from $\mathcal{K}$ to $\mathcal{H}$ such that the series $\sum_{j} V_{j}^{*} a V_{j}$ converge strongly.

Proof. Clearly a completely positive map of the form (2.14) is normal. Indeed, for every non-decreasing net $\left(x_{\alpha}\right)_{\alpha}$ of positive elements of $\mathcal{A}$ converging strongly to its least upper bound $x$ we have

$$
\begin{aligned}
\sup _{\alpha}\left\langle u, T\left(x_{\alpha}\right) u\right\rangle & =\sum_{j} \sup _{\alpha}\left\langle V_{j} u, x_{\alpha} V_{j} u\right\rangle \\
& =\sum_{j}\left\langle V_{j} u, x V_{j} u\right\rangle \\
& =\langle u, T(x) u\rangle
\end{aligned}
$$

for every $u \in \mathcal{K}$.

Conversely we can represent the normal completely positive map $T$ in the form $T(a)=V^{*} \pi(a) V$ with $\pi$ normal as in Lemma 2.19. Therefore it suffices to establish (2.14) for the representation $\pi$. By decomposing $\mathcal{K}_{1}$ into cyclic orthogonal subspaces we can suppose that there exists a cyclic vector $w$ for $\pi(\mathcal{A})$ in $\mathcal{K}_{1}$. The state on $\mathcal{A}$

$$
a \rightarrow\langle w, \pi(a) w\rangle
$$

is normal because $\pi$ is normal. Hence (see, for example, [21] Th. 2.4.21 p.76) there exists a sequence $\left(u_{n}\right)_{n \geq 1}$ of vectors in $\mathcal{H}$ such that

$$
\sum_{n \geq 1}\left\|u_{n}\right\|^{2}=1, \quad\langle w, \pi(a) w\rangle=\sum_{n \geq 1}\left\langle u_{n}, a u_{n}\right\rangle
$$


for all $a \in \mathcal{A}$. Moreover, for every $x \in \mathcal{A}$ and $n \geq 1$, we have

$$
\left\|x u_{n}\right\|^{2}=\left\langle u_{n},\left(x^{*} x\right) u_{n}\right\rangle \leq\left\langle w, \pi\left(x^{*} x\right) w\right\rangle=\|\pi(x) w\|^{2} .
$$

Therefore there exist contractions $V_{n}: \mathcal{K}_{1} \rightarrow \mathcal{K}$ such that

$$
V_{n} \pi(x) w=x u_{n} \text {. }
$$

For all $x \in \mathcal{A}$ we have also

$$
\begin{aligned}
\langle\pi(x) w, \pi(a) \pi(x) w\rangle & =\left\langle w, \pi\left(x^{*} a x\right) w\right\rangle \\
& =\sum_{j=1}^{\infty}\left\langle u_{j}, x^{*} a x u_{j}\right\rangle \\
& =\sum_{j=1}^{\infty}\left\langle x u_{j}, a x u_{j}\right\rangle \\
& =\sum_{j=1}^{\infty}\left\langle V_{j} \pi(x) w, a V_{j} \pi(x) w\right\rangle \\
& =\sum_{j=1}^{\infty}\left\langle\pi(x) w, V_{j}^{*} a V_{j} \pi(x) w\right\rangle
\end{aligned}
$$

This completes the proof because $w$ is cyclic for $\mathcal{A}$.

Remark. It is worth noticing here that Kraus' representation (2.14) can also be written in the form

$$
T(a)=V^{*}(a \otimes \mathbb{1}) V
$$

where $\mathbb{1 l}$ denotes the identity operator in another Hilbert space $\mathcal{H}_{1}$ and $V: \mathcal{K} \rightarrow$ $\mathcal{H} \otimes \mathcal{H}_{1}$ is a bounded operator. In fact it suffices to consider an orthonormal basis $\left(e_{j}\right)$ in $\mathcal{H}_{1}$ and define

$$
V u=\left(V_{j} u\right) \otimes e_{j}
$$

The notion of minimality for Kraus representations is obviously a special case of the analogue for Stinespring representation.

\subsection{A quantum Feynman-Kac formula}

In this section we outline a perturbation technique similar to the FeynmanKac perturbation in classical probability. The abstract algebraic generalisation described by Accardi in [1] shows the importance of the notion of cocycle in the construction of algebraic Markov processes.

We shall use the notation of Section 2.1. $(\mathcal{A}, \varphi)$ is an algebraic probability space with a filtration $\left(\mathcal{A}_{t]}\right)_{t \geq 0}$; conditional expectations $\mathbb{E}\left[\cdot \mid \mathcal{A}_{t]}\right]$ will be denoted also by $\mathbb{E}_{t]}$. 
Definition 2.21 A family $\left(\theta_{t}\right)_{t \geq 0}$ of $*$-homomorphisms of $\mathcal{A}$ is called a covariant shift if:

1. (semigroup property) $\theta_{0}(a)=a$ and $\theta_{t}\left(\theta_{s}(a)\right)=\theta_{t+s}(a)$ for all $s, t \geq 0$ and all $a \in \mathcal{A}$,

2. (left inverse) for all $t \geq 0$ the map $\theta_{t}$ has a left inverse denoted $\theta_{t}^{*}$, i.e. for all $a \in \mathcal{A}$, we have

$$
\theta_{t}^{*}\left(\theta_{t}(a)\right)=a,
$$

3. (covariance) for all $s, t \geq 0$ and all $a \in \mathcal{A}$ we have

$$
\theta_{t}\left(\mathbb{E}\left[\theta_{s}(a) \mid \mathcal{A}_{0 !}\right]\right)=\mathbb{E}\left[\theta_{t+v}(a) \mid \mathcal{A}_{t]}\right] .
$$

It is easy to check that the standard time shift of classical homogencous Markov process is a covariant shift according to the above definition. In fact, with the notation of Example 2.1, is defined by

$$
\theta_{t}\left(f\left(x_{t_{1}}, \ldots, x_{t_{n}}\right)\right)=f\left(x_{t+t_{1}}, \ldots, x_{\ell+t_{n}}\right) .
$$

Consider a family $\left(j_{l}\right)_{t \geq 0}$ of $*$-homomorp! lisms on $\mathcal{A}$ which are adapted in the sense that

$$
j_{t}\left(\mathbb{E}\left[a \mid \mathcal{A}_{t]}\right]\right)=\mathbb{E}\left[j_{t}(a) \mid \mathcal{A}_{t]}\right]
$$

for every $a \in \mathcal{A}$. We now try to find conditions in order that the algebraic process $u$ on $(\mathcal{A}, \varphi)$ with values in $\mathcal{A}_{0]}$ defined by

$$
u_{t}(\cdot)=j_{t}\left(\theta_{t}(\cdot)\right)
$$

is a covariant algebraic Markov process.

The following proposition gives a necessary condition.

Proposition 2.22 Let $\left(j_{t}\right)_{t>0}$ be a family of $*$-homomorphisms on $\mathcal{A}$ satisfying condition (2.15). Suppose that the algebraic process $u$ defined by (2.16) is a covariant algebraic Markov process. Then, for all $s, t \geq 0$ and all $a \in \theta_{t+s}\left(\mathcal{A}_{0]}\right)$ we have

$$
\mathbb{E}_{0]}\left[j_{t+s}(a)\right]=\mathbb{E}_{0\}}\left[j_{t}\left(\theta_{t}\left(j_{s}\left(\theta_{t}^{*}(a)\right)\right)\right)\right] .
$$

Proof. If $u$ is a covariant quantum Markov process then, by Proposition 2.4, the family $\mathcal{T}$ of linear maps defined by (2.3) is a semigroup on $\mathcal{A}_{00}$. Now, for all $s, t \geq 0$ and all $a \in \mathcal{A}_{01}$, denoting the conditional expectation with respect to $\mathcal{A}_{s]}$ by $\mathbb{E}_{s]}$, we have

$$
\begin{aligned}
\mathcal{T}_{t}\left(\mathcal{T}_{s}(a)\right) & =\mathbb{E}_{0 !}\left[j_{t}\left(\theta_{t}\left(\mathbb{E}_{0 !}\left[j_{s}\left(\theta_{s}(a)\right)\right]\right)\right)\right] \\
\text { covariance } & =\mathbb{E}_{0]}\left[j_{t}\left(\mathbb{E}_{t]}\left[\theta_{t}\left(j_{s}\left(\theta_{s}(a)\right)\right)\right]\right)\right] \\
j \text { is adapted } & =\mathbb{E}_{0]}\left[\mathbb{E}_{t]}\left[j_{t}\left(\theta_{t}\left(j_{s}\left(\theta_{s}(a)\right)\right)\right)\right]\right] \\
\text { projectivity of } \mathbb{E} & =\mathbb{E}_{0\}}\left[j_{t}\left(\theta_{t}\left(j_{s}\left(\theta_{s}(a)\right)\right)\right)\right] \\
\text { inverse \& semigroup } & =\mathbb{E}_{0 !}\left[j_{t}\left(\theta_{t} \circ j_{s} \circ \theta_{t}^{*}\right)\left(\theta_{t+s}(a)\right)\right]
\end{aligned}
$$


On the other hand $\mathcal{T}_{t+s}$ is defined by

$$
\mathcal{T}_{t+s}(a)=\mathbb{E}_{0]}\left[j_{t+s}\left(\theta_{t+s}(a)\right)\right]
$$

for all $a \in \mathcal{A}_{0]}$. Therefore, since $\mathcal{T}$ is a semigroup, 2.17 holds.

Definition 2.23 A family $\left(j_{t}\right)_{t \geq 0}$ of $*$-homomorphisms on $\mathcal{A}$ satisfying (2.15) is called a Markov cocycle with respect to the covariant shift $\theta$ if, for all $s, t \geq 0$ and all $a \in \mathcal{A}_{0]}$, we have

$$
j_{t+s}\left(\theta_{t+s}(a)\right)=j_{t}\left(\theta_{t}\left(j_{s}\left(\theta_{s}(a)\right)\right)\right) .
$$

We prove now the fundamental result of [1].

Theorem 2.24 Let $\theta$ be a covariant shift and let $\left(j_{t}\right)_{t \geq 0}$ be a Markov cocycle with respect to $\theta$. Then the algebraic process $u$ defined $\bar{b} y(2.16)$ is a covariant algebraic Markov process on $(\mathcal{A}, \varphi)$ with values in $\mathcal{A}_{0]}$.

Proof. The proof of Proposition 2.22 shows that the maps $\left(\mathcal{T}_{t}\right)_{t \geq 0}$ are a semigroup. Let us show first that $u$ is a quantum Markov process. For all $a \in \mathcal{A}_{0}$ ] and all $s, t \geq 0$ we have

$$
\begin{aligned}
\mathbb{E}_{s]}\left[u_{t+s}(a)\right] & =\mathbb{E}_{s]}\left[j_{t+s}\left(\theta_{t+s}(a)\right)\right] \\
\text { cocycle property } & =\mathbb{E}_{s]}\left[j_{s}\left(\left(\theta_{s} \circ j_{t} \circ \theta_{s}^{*}\right) \theta_{t+s}(a)\right)\right] \\
\text { projectivity of } \mathbb{E} & =\mathbb{E}_{s]}\left[j_{s}\left(\mathbb{E}_{s]}\left[\left(\theta_{s} \circ j_{t} \circ \theta_{s}^{*}\right) \theta_{t+s}(a)\right]\right)\right] \\
\text { covariance of } \theta \text { and } \mathbb{E} & \left.=\mathbb{E}_{s]}\left[\left(j_{s} \circ \theta_{s}\right)\left(\mathbb{E}_{0]}\left[j_{t}\left(\theta_{t}(a)\right)\right)\right]\right)\right] \\
& \left.=\mathbb{E}_{s]}\left[\left(j_{s} \circ \theta_{s}\right)\left(\mathcal{T}_{t}(a)\right)\right]\right] \\
& =u_{s}\left(\mathcal{T}_{t}(a)\right)
\end{aligned}
$$

Let us show now that $u$ is covariant; for all $a \in \mathcal{A}_{0]}$ and all $s, t \geq 0$, we have

$$
u_{t}\left(\mathbb{E}_{0]}\left[u_{s}(a)\right]\right)=u_{t}\left(\mathcal{T}_{s}(a)\right)=\mathbb{E}_{t]}\left[u_{t+s}(a)\right]
$$

This completes the proof.

Remark. The analogy with the classical Feynman-Kac formula becomes clear if we take a standard Brownian motion $\left(w_{t}\right)_{t \geq 0}$ with natural filtration $\left(\mathcal{F}_{t}\right)_{t \geq 0}$

$$
\mathcal{F}_{t}=\sigma\left\{w_{s} \mid 0 \leq s \leq t\right\}
$$

as the classical stochastic process $x$ in Example 2.1. Let $M_{t}$ be the multiplicative functionals

and let

$$
M_{t}=\exp \left(-\int_{0}^{t} c\left(w_{s}\right) d s\right)
$$

$$
j_{t}(a)=M_{t} a .
$$

Clearly $j_{t}: \mathcal{A} \rightarrow \mathcal{A}$ is not an identity preserving homomorphism but it enjoys the cocycle property (2.18) with respect to the standard (classical) shift $\theta$ (see [2]). 
Proposition 2.25 Let $\mathcal{H}$ be a complex separable Hilbert space, let $\mathcal{A}$ be the *-algebra $\mathcal{B}(\mathcal{H})$ of all bounded operators on $\mathcal{H}$ and let $\left(\mathcal{A}_{t]}\right)_{t \geq 0}$ a filtration of sub- ${ }^{*}$-algebras of $\mathcal{A}$. Consider a family $\left(V_{t}\right)_{t \geq 0}$ of unitary operators on $\mathcal{H}$ such that $V_{t} \in \mathcal{A}_{t]}$ and, for all $t \geq 0$, define the map

$$
j_{t}: \mathcal{A} \rightarrow \mathcal{A}, \quad j_{t}(a)=V_{t} a V_{t}^{*}
$$

Suppose that, for all $s, t \geq 0$, we have

$$
V_{t+s}=V_{t} \theta_{t}\left(V_{s}\right)
$$

Then $j$ is a cocycle with respect to $\theta$.

Proof. For all $s, t \geq 0$ and all $a \in \mathcal{A}_{0\}}$ we have

$$
\begin{aligned}
j_{t} \circ \theta_{t} \circ j_{s} \circ \theta_{s}(a) & =V_{t}\left(\theta_{t}\left(V_{s} \theta_{s}(a) V_{s}^{*}\right)\right) V_{t}^{*} \\
& =\left[V_{t} \theta_{t}\left(V_{s}\right)\right] \theta_{t+s}(a)\left[V_{t} \theta_{t}\left(V_{s}\right)\right]^{*} \\
& =j_{t+s}\left(\theta_{t+s}(a)\right)
\end{aligned}
$$

as required.

The family of operators $\left(V_{t}\right)_{t \geq 0}$ defining the cocycle $j$ is called an operator cocycle or, when no confusion can arise, simply a cocycle. 